\title{
La contaminación en la tradición del Chevalier de la Charrette: el caso del manuscrito A*
}

\author{
Carlo Beretta \\ Universidad de la Basilicata
}

Título: La contaminación en la tradición del Chevalier de la Charrette: el caso del manuscrito A.

Resumen: A partir de la edición del Chevalier de la Charrette de Chrétien de Troyes, en el haber Wendelin Foerster, y del examen del corpus manuscrito de esta obra por parte de Alexandre Micha, el presente artículo, escrito desde los presupuestos de la crítica neolachmanniana, saca a la luz varios casos de contaminación, incidiendo sobre todo en las relaciones del llamado manuscrito $A$ con el resto de la tradición.
Title: The Contamination in the Tradition of Chevalier de la Charrette: the Case of the Manuscript A.

Abstract: In view of the edition of the Chevalier de la Charrette by Chrétien de Troyes, in the work of Wendelin Foerster, and the evaluation of the manuscript corpus of this poem by Alexandre Micha, this article, written from the neolachmannian criticism, explains several cases of contamination, focusing especially on the relations of the so-called manuscript $A$ with the rest of the tradition.

Key words: Chevalier de la Charrette, Chrétien de Troyes, Contamination, Manuscript $A$.

Date of Receipt: 11/1/2016.
Date of Approval: 24/2/2016.
Fecha de aceptación: 24/2/2016.

Palabras clave: Chevalier de la Charrette, Chrétien de Troyes, Contaminación, Manuscrito $A$.

Fecha de recepción: 11/1/2016.

La única edición del Chevalier de la Charrette de Chrétien de Troyes que puede presumir de la etiqueta de "crítica" sigue siendo, a pesar del paso del tiempo, la de Wendelin Foerster, cuarto volumen de los Sämtliche

Traducción de Francisco José Rodríguez Mesa (Universidad de Córdoba). 
erhaltene $W_{e r k e}$. Todos los testimonios hasta entonces conocidos fueron confrontados, se trazó un stemma codicum y se preparó un aparato crítico que, si ante un examen profundo revela no pocas omisiones e imprecisiones, continúa siendo el único disponible hoy día. Las notas al texto abordan con la pericia habitual de Foerster numerosos pasajes de controvertida interpretación (desgraciadamente, no todos); algunas de ellas constituyen pequeñas monografías lingüísticas que todavía hoy son dignas de admiración. Ninguna de las ediciones posteriores, casi todas basadas en criterios bédierianos y, fundamentalmente, en la copia de Guiot, revisada y corregida, alcanza el nivel de la vieja edición alemana. El examen de la tradición manuscrita de las obras de Chrétien fue retomado, tiempo después de Foerster, por Alexandre Micha ${ }^{3}$ en plena estación bédierista: es significativo que este amplio estudio, si bien basado en el método de los errores comunes, no haya producido ninguna edición de corte lachmanniano, convirtiéndose en letra muerta ${ }^{4}$. Entre las más recientes destaca la de Alfred Foulet y Karl D. Uitti. Los dos editores, basándose en una serie de criterios genéricos ${ }^{5}$ enumerados en algunos artículos preliminares, publicaron una edición cuyo texto coincide en gran medida con el de Foerster ${ }^{6}$.

Los testimonios del Chevalier de la Charrette conocidos por Foerster son los siguientes:

C: París, BNF, fr. 794 (es la famosa copia de Guiot): texto completo.

2 Christian von Troyes, Sämtliche erhaltene Werke, nach allen bekannten Handschriften herausgegeben von Wendelin Foerster; Vierter Band: Karrenritter und Wilhelmsleben, Halle, Niemeyer, 1899. De esta edición se adopta la numeración de los versos.

3 Alexandre Micha, La tradition manuscrite des romans de Chrétien de Troyes, Genève, Droz, $1966^{2}$ (si bien la obra es de 1939); al Chevalier de la Charrette se dedican las pp. 128-145.

4 Micha publicará una edición bédieriana del Cligés, aparecida en la serie Les Romans de Chrétien de Troyes édités d'après la copie de Guiot, al cuidado, en los Classiques Français du Moyen Âge de la editorial Champion, del bédieriano Mario Roques.

5 Se trata de criterios obvios (como, por ejemplo, el usus scribendi), utilizados también por los editores lachmannianos, pero en un segundo estadio, en el nivel de la constitutio textus.

6 Chrétien de Troyes, Le Chevalier de la Charrette (Lancelot), eds. Alfred Foulet et Karl D. Uitti, Paris, Bordas (Classiques Garnier), 1989. Para los artículos preliminares remitimos a la bibliografía de la edición, pp. XLII-XLIII. 
T: París, BNF, fr. 12560: texto completo.

V: Ciudad del Vaticano, Biblioteca Apostólica Vaticana, Reg. Lat. 1725: acéfalo, el texto comienza en el v. 861 de la edición de Foerster. A: Chantilly, Musée Condé, 472: mútilo, el texto termina en el v. 5873 de la edición de Foerster, poco antes de la mitad de la primera columna del folio $213 \mathrm{v}^{7}$; además, también falta el prólogo (vv. 1-30). E: Escorial, Real Biblioteca del Monasterio, M. III. 21: mútilo, el texto concluye en el v. 5763 de la edición de Foerster.

F: París, BNF, fr. 1450: acéfalo, el texto comienza en el v. 5652 de la edición de Foerster.

Otros dos testimonios permanecieron desconocidos para Foerster:

I: Paris, Bibliothèque de l'Institut de France, 6138: fragmento de códice que contiene, entre otros materiales, los vv. 3615-54, 3735-74 y 4741-899 de la edición de Foerster.

$G$ : Princeton, University Library, Garrett 125: códice fragmentario, encuadernado en modo desordenado que contiene, entre otros materiales, los vv. 1-290; 961-1304, 1473-642, 2318-469, 2642-987, 3640-976 de la edición de Foerster.

A estos manuscritos se debe añadir un dérimage, contenido en algunos testimonios del Lancelot en prosa, editado recientemente en dos ocasio$n^{8}{ }^{8}$, y desgraciadamente, aunque no siempre, poco útil (sigla $V D$ ).

Hay ocho testimonios, de los cuales solo dos $(C$ y $T)$ están relativamente completos.

El manuscrito $A$, del que nos ocuparemos aquí con empeño, es una amplia colección de romans en verso y en prosa ${ }^{9}$, fechables en torno a la mitad del siglo XIII; probablemente proviene, habida cuenta de los rasgos lingüísticos, del área de Flandes-Hainaut. El Chevalier de la Charrette está contenido entre los folios $196-213 \mathrm{v}^{10}$. A es testimonio también de Erec et

$7 \quad$ El resto del folio está en blanco.

8 David F. Hult, "Le Conte de la Charrette: version dérimée du Chevalier de la charrette de Chrétien de Troyes", Romance Philology, LVII (2004), pp. 127-322; Le “Conte de la charrette" dans le "Lancelot" en prose: une version divergente de la Vulgate, ed. Annie Combes, Paris, Champion, 2009. Se cita por esta última edición.

9 De algunos de los cuales es testimonio único.

10 Según la numeración actual. En realidad, se trataría del fol. 214: por error, dos 
Enide y del Chevalier au Lion ${ }^{11}$. Valga lo que valiere, el juicio de Micha sobre el texto de la Charrette copiado en $A$ no es del todo desfavorable: "Sans être un bon ms., il est certainement moins retouché que le $B . N$. 1450, le ms. du Vatican ou le ms. de l'Escurial"12; "Ce ms. de Chantilly, qui est loin d'être le plus modifié, a traité son modèle avec un peu plus de respect, mais autant de maladresse que ses frères, les mss. du Vatican et de l'Escurial"13.

Foerster propuso el siguiente stemma codicum:

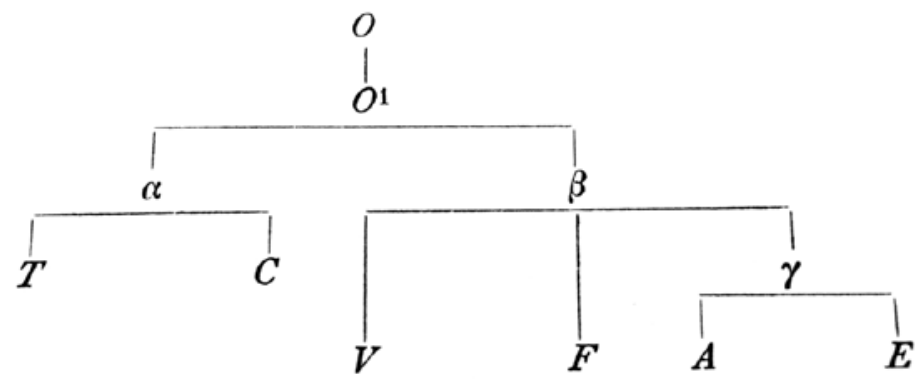

Sin embargo, el estudioso alemán no aporta prueba alguna que sostenga su reconstrucción; un poco (;sic!) "der Raumesparnis wegen”, pero también (y sobre todo) por una cierta desconfianza operativa en su stemma ${ }^{14}$, que no

folios seguidos llevan la numeración 203.

11 Sobre los manuscritos de Chrétien de Troyes es fundamental la obra colectiva Les Manuscrits de Chrétien de Troyes / The Manuscripts of Chrétien de Troyes, eds. Keith Busby, Terry Nixon, Alison Stones, Lori Walters, Amsterdam-Atlanta, Rodopi, 1993, 2 vols. La descripción pormenorizada del manuscrito de Chantilly, con bibliografía, se encuentra en el vol. II, pp. 39-41. Cf. también Alexandre Micha, op. cit., pp. 38-39, quien llega a distinguir siete manos distintas; la cuarta sería la responsable de la transcripción de la Charrette. Una caracterización del contenido del testimonio, precioso transmisor de unica, epígonos de Chrétien de Troyes, en Keith Busby, Codex and Context. Reading Old French Verse Narrative in Manuscript, Amsterdam-New York, Rodopi, 2002, pp. 405-13 (y también pp. 87-93). La reproducción fotográfica de los testimonios de la Charrette (salvo el fragmento I) está disponible en la web http://www.princeton.edu/ lancelot/new-traditional/.

12 Alexandre Micha, op. cit., p. 346.

13 Ibidem, p. 348.

14 También en las tres ediciones anteriores, Cligés, Chevalier au Lion ed Erec et Eni$d e$, Foerster no se muestra generoso en demostraciones y alberga no pocas reservas 
parece limitarse al caso concreto del Chevalier de la Charrette: "Denn eine solche [scil. Stammbaum] ist, mag sie vollständig oder reich oder nur summarisch sein, doch ohne jeden praktischen Nutzen" ${ }^{15}$. Entre los motivos de esta desconfianza uno de los principales es la práctica de contaminación y conjetural de los copistas, que oscurece, cambia y a menudo impide el establecimiento de vínculos entre los testimonios ${ }^{16}$. De tales prácticas el editor no aporta ni tan siquiera un ejemplo relativo al Chevalier de la Charrette, y su stemma está desprovisto de indicaciones en esta dirección. Además, observa Foerster, también un stemma insostenible, acompañado de las pruebas recogidas por el editor, llevará a quien confíe en él a las mismas y discutibles conclusiones. El único sistema para probar la validez de un stemma consiste en dejar a un lado el que otros hayan trazado y construir uno basado en la misma varia lectio ${ }^{17}$. De cualquier forma, concluye el editor, dicho estema es el que "der der grössten Zahl der Einzelnfälle gerecht wird" ${ }^{18}$. Foerster, poco dado al esprit de système, no confirió a sus parcas - aunque agudas y reiteradas - consideraciones un alcance teórico; estas quedaron confinadas en las pocas páginas de las introducciones dedicadas a los vínculos entre los manuscritos ${ }^{19}$. En efecto, su constitutio textus deja entrever el escepticismo expresado en la introducción. A menudo se siente libre para colocar en el texto la lección que más le satisface, incluso contraviniendo los datos de su propio stemma. Así, si bien afirma privilegiar el manuscrito $\mathrm{T}^{20}$, su praxis es en buena medida libre.

Un control sobre toda la tradición manuscrita del Chevalier de la Charrette, con vista puesta en una nueva edición crítica, según el deseo del mismo Foerster, me ha permitido concluir que, a grandes rasgos, el stemma de Foerster supera la prueba. No procedo a demostrarlo, puesto que se

sobre la eficacia operativa de sus stemmata. El Conte du Graal se publicará, como último volumen de las Sämtliche Werke, solo en 1932, por Alfons Hilka.

15 Christian von Troyes, op. cit., p. VIII.

16 Ibidem, p. VI.

17 Ibidem, p. VIII.

18 Ibidem, p. IX.

19 En nuestro caso, solo algo más de cinco páginas: desde p. VI a p. XI.

20 "Im grossen und ganzen verdient $T$ die meiste Rücksicht uns steht deshalb oft gegen alle übrigen im Text"; aunque "oft lässt auch er im Stich": Christian von Troyes, op. cit., p. XI. Para la lengua, en cambio, tanto en estas como en las otras ediciones de Chrétien, Foerster se basa sobre todo en $C$. 
trata de un tema ajeno al de este estudio; sin embargo, puedo afirmar que, más allá de la existencia del arquetipo $O^{1}$, de la cual se pueden ofrecer pruebas certeras, importantes convergencias confirman la distribución de los testimonios en dos familias, $\alpha(C T)$ y $\beta(V A E F)^{21}$. La rama $\beta$, sobre todo, se define sobre la base de numerosos errores y alteraciones comunes a sus miembros. Menos clara es la situación de $\alpha$ : $C$ y $T$ están vinculados por un número marcadamente inferior de errores, pero estos son de una naturaleza tal —a mi juicio- que no dejan lugar a dudas. Su escaso número se podría atribuir a la calidad de la lección de su modelo. No obstante, es digno de mención que Foerster dudase largo tiempo entre el stemma que consideraba más verosímil y otro alternativo, que concebía $C$ y $T$ aislados y opuestos a $\beta^{22}$. Este hecho debe considerarse, cuando menos, un punto de partida relativamente sólido.

Notablemente diverso se muestra el stemma establecido por Micha tras una demostración repleta, por desgracia, de numerosos errores materia$\operatorname{les}^{23}$ y de método ${ }^{24}$ :

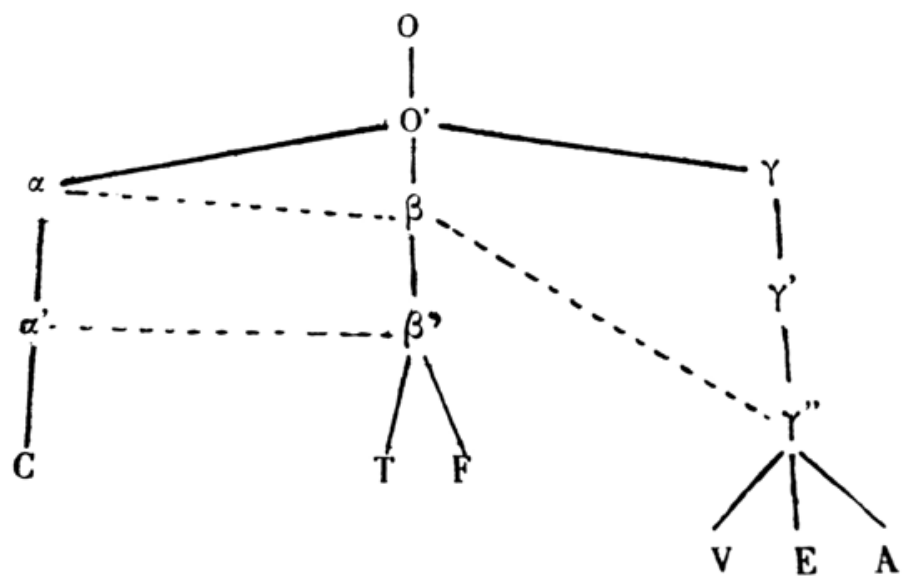

21 Aún hay que evaluar la posición de G y, en parte, de F. No es necesario tomar en consideración los escasos versos conservados en $I$.

22 Christian von Troyes, op. cit., p. X.

23 Debidos, en su mayoría, a que Micha siguió incautamente el aparato crítico de Foerster, como atestiguan los numerosos errores comunes.

24 Alexandre Micha, op. cit., p. 145. Micha no recoge los pocos, pero decisivos, errores comunes a $\mathrm{C}$ y $\mathrm{T}$. 
Aquí, en cambio, vemos representados gráficamente algunos vínculos "horizontales", cuyos términos el estudioso resume afirmando "les lignes $\alpha \beta, \beta \gamma^{\prime}$, $\alpha^{\prime} \beta^{\prime}$ montrent pourquoi nous relevons par exemples des accords $C A$, ou $T V$ ou $T A E^{\prime 25}$. El problema radica en que la definición de estos vínculos es demasiado genérica, habiéndose trazado las líneas aludidas no de testimonio a testimonio, o de testimonio a subarquetipo, sino únicamente de subarquetipo a subarquetipo. En efecto, concluye el propio Micha:

Pour établir ce stemma idéal [que tenga en cuenta todos los datos disponibles], non seulement trop d'intermédiaires nous manquent, mais il serait nécessaire de savoir combien de mss. ont pratiqué ces amalgames passagers, et de combien d'amalgames chaque ms. est le résultat ${ }^{26}$.

El problema de la contaminación, que Foerster observó insistentemente, aunque no llegó a demostrar, fue tratado de manera más difusa por Micha, si bien este no indicó en su esquema los vínculos que unen de modo individual los distintos testimonios. Sin embargo, enumera una serie de exactos acuerdos "transversales" que, en ocasiones, se someten a un exhaustivo análisis.

Estudiando por mi cuenta la tradición del Chevalier de la Charrette, me he encontrado con al menos dos casos de segura contaminación por parte de un testimonio que tienen que ver con los manuscritos $E$ y $A$. Querría examinar aquí el caso de $A^{27}$.

25 Ibidem.

26 Ibidem.

27 Por lo que respecta a $E$, contamos con dos estudios de Lenora D. Wolfgang, "The Manuscripts of the Chevalier de la Charrette (Lancelot) of Chrétien de Troyes. Preliminary Remarks to a New Edition: the Case of ms. E", en Miscellanea Mediaevalia. Mélanges offerts à Philippe Ménard, eds. Jean-Claude Faucon, Alain Labbé, Danielle Quéruel, Paris, Champion, 1998, II, pp. 1487-1488; "The Manuscripts of the Chevalier de la Charrette (Lancelot) of Chrétien de Troyes: Preliminary Remarks to a New Edition: The Case of ms. E Part II", en "De sens rassis". Essays in honor of Rupert T. Pickens, eds. Keith Busby, Bernard Guidot, Logan E. Whalen, Amsterdam-New York, Rodopi, 2005, pp. 729-753. Estos son de escasa utilidad, puesto que se limitan a examinar una por una las numerosas lagunas del manuscrito, tratando de identificar las causas, pero sin evaluarlas a la luz del grueso de la tradición. 
$A$ comparte buena parte de los errores, de las lecciones inferiores, pero no claramente erróneas, y de las características de $\beta$. Además, en ocasiones concuerda con los errores solo de $E$, lo que permitió a Foerster conjeturar la existencia del subarquetipo $\gamma$, descendiente de $\beta$. Sin embargo, el testimonio parece abandonar muy frecuentemente la lección de su familia para adoptar la de $\alpha$ o alguno de sus derivados; en ocasiones uniéndose a $C$, en otras a $T$ y en otras a ambos, en oposición a $\beta$.

La actividad de contaminación de $A$ es observable sin lugar a dudas al menos en un caso altamente significativo. En los vv. 5406-08 Foerster coloca en el texto: "[...] et si le [el rey] tindrent | Mout an grant, qu'un don lor donast | Et lor voloir lor otreast”. En el v. 5407 la lección acogida es la de $C T(\alpha)$, mientras en $\beta(V A E)$ se lee "que il lor donast". En el v. 5408 se mantiene el texto de $\alpha$; $V E$ recogen, coherentes con la lección del verso anterior, "I. don et si ( $E$ et qu'il) lor otroiast"; $A$ se separa de $\beta$, y adopta en este verso la lección de $\alpha$ : "Et lor voloir lor otriast". La frase que de aquí se deriva carece de sentido: "Molt en grant que il lor donnast |Et lor voloir lor otriast": desaparece la mención al "don", sin la cual el pasaje no se entiende, y "donnast" se queda sin objeto. La mecánica yuxtaposición de dos redacciones diferentes, con el efecto de convertir el pasaje en algo incomprensible y sintácticamente inaceptable demuestra, a las claras, que $A$ tenía a su disposición al menos dos ejemplares, uno de $\beta$ y uno de $\alpha$. Es hipotetizable que el recurso a dos fuentes distintas se deba a una laguna del ejemplar base, probablemente un verso perdido ${ }^{28}$.

Con un grado de fiabilidad inferior, pero también digno de mención, es el v. 3040: $C T$ "De chasque ( $T$ chascune) part ot .i. grant ( $T$ ot: -1$)$ tronc" $\| V E$ "Dambes .ii. parz avoit .i. tronc". $A$ parece haberse entrecruzado (de forma errónea) con las dos lecciones equivalentes en $\alpha(C T)^{29}$ y $\beta(V E)$ : "Quasqune part avoit .i. tronc"; en la segunda mitad del v. se lee, como en $\beta$ ("avoit un"); en la primera mitad se acerca en cambio a $\alpha$ ("Quasqune"), pero la omisión de "De" es forzosa para evitar la conse-

28 Micha cita este pasaje a colación de un posible reagrupamiento $V E$ (Alexandre Micha, op. cit., p. 130). Pero aquí $V E=\beta$.

29 Que se puede reconstruir "De chascune part ot un tronc", donde $C$ sustituye "chascune" por "chasque", que parece ajeno a la lengua del autor; "grant" sirve solo para compensar la pérdida de una sílaba; $T$ se limita a omitir el numeral (escrito .i. en el ejemplar). Foerster se acoge a la lección de $\alpha$. 
cuente hipermetría ${ }^{30}$.

Correspondencias no banales de $A$ con $C T(=\alpha)$, opuestas a $\beta$ en la lección probablemente original, o variante adiáfora, o lección inferior, pero no manifiestamente errónea, se hallan a lo largo de casi todo el texto ${ }^{31}$ :

v. 903: $C T A$ "si fera voir" || VE "non fera voir". Original.

v. 1347: $C T A$ "Pansers li plest parlers ( $T$ pensers $A$ penser) li grieve" $\|$ $V E$ "Penser li fet amors et grieve". La lección de $C$ es sin duda la genuina; sobre esta $T$ y $A$ han injertado independientemente un error de repetición ("pensers [...] pensers"). Original.

v. 1463: CTA “de voir dire gueitiee" $\| V E$ "devant tres bien gueitiee”. Original.

v. 1550: $C T A$ "po ( $A$ molt poi) m'an chaut" $\| V E G$ "or ( $E$ om. $)$ ne vos chaut". Original.

v. 1552: $C T A$ “que dite m'aiez” ( $A$ “m'avez”) \|VEG “que veüe ( $E$ veü $G$ vos veü) aiez”. Original.

v. 4297: $C T A$ "trestote prise" || $V E$ "ja tote prise". Adiáfora.

v. 4299: $C T A$ "Donc fera ele" \|VE "Ne ( $E$ Donc ne) fera el”. Probablemente $C T A$ contienen una lección inferior ${ }^{32}$. Nótese que $E$ (hipermetro) parece cruzar las lecciones de CTA y $V$.

v. 4627: $C T A$ "Rien fors vos" || $V E$ "Rienz nule". Adiáfora.

v. 4659: $C T A$ "La premiere ongle" (TA “once") || VE "La premiere jointe". "Once" es sin duda la lección genuina ${ }^{33}$; $C$ banaliza, pero claramente partiendo de "once"; $V E$ adoptan otra trivialización. No se puede excluir esta vez que $V E$ hayan banalizado independientemente.

En dos ocasiones, al menos, $A$ se une a $C T$ contra $\beta$ en errores manifiestos, pero en ambos casos el error podría ser poligenético.

v. 1940: CTA "Dom estes vos et de quel leu" $\| V E$ "Qui estes vos et

30 Cabe la duda de que $A$ use aquí una construcción sin preposición, atestiguada con la palabra "part" (pero no, por lo que parece, con "chascun"), del tipo de "totes parz", en lugar de "de totes parz". Cf. Adolf Tobler, Erhard Lommatzsch, Altfranzösisches Wörterbuch, VII, columnas 359-67, s.v. part.

31 Aquí, como en el resto de casos, cuando se cita una lección común a dos o más manuscritos, la grafía será la del manuscrito citado en primer lugar, con las normales actualizaciones ( $u>v ; i>j ; c+a, o, u>c$; división de las palabras; mayúsculas). Los diferentes reagrupamientos se separan con dos barras verticales $\|$.

32 La pregunta retórica a la que apuntan $V E$ (Ne fera el(e) [...]?) parece difficilior.

33 Para once, véase la nota de Foerster: Christian von Troyes, op. cit., p. 402. 
de quel lieu". "Dom" es claramente absurdo, puesto que, equivaliendo a "de quel leu", introduce una tautología. Pero el mismo error aparece, a cargo solo de $C$, en el Conte du Graal, v. 5595: "Dom il ert", en lugar del correcto "Qui il ert" ${ }^{4}$. La poligénesis no se puede excluir.

v. 2219: CTA ".I. chevalier sor ( $T$ souz) la bretesche" \|VE "Un chevalier de la bretesche". La lección de $C A$, de la que también proviene la fallida tentativa de $T$ de ajustar el sentido ("souz" en lugar de "sor"), es absurda: el caballero hace una incursión "desde la" bretesche para luchar contra los adversarios: ¿qué se supone que iba a hacer (para más inri, a caballo) "encima de la" bretesche? Pero los vv. 2215 ("Cil qui sor la bretesche

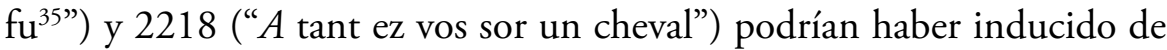
manera independiente al error de $C T A^{36}$.

No es verosímil que un copista extraiga de un ejemplar de control un gran número de errores manifiestos ${ }^{37}$. La fenomenología de los casos enumerados, entre los que destaca la ausencia de coincidencias significativas entre $A$ y $\alpha$ por lo que atańe a los errores, debería ser suficiente para demostrar la actividad de contaminación de $A^{38}$. Por otra parte, que el ejemplar base de $A$ pertenecía a $\beta$ puede demostrarse por la convergencia de nuestro manuscrito con $V E F G^{39}$ en un número de lecciones erróneas mucho más certeras y abundantes que las que lo vinculan a $C T^{40}$. Doy de ello una breve lista, en aras de la brevedad, desprovista de comentarios ${ }^{41}$. Errores comunes a VAE: vv. 1125, 3318, 4210, 4561, 5305, 5552. Erro-

34 Chrétien de Troyes, Le Roman de Perceval ou Le Conte du Graal, ed. Keith Busby, Tübingen, Niemeyer, 1993, p. 226.

35 Donde, además, no se habla del caballero, sino de una atalaya situada, precisamente, "sor la bretesche".

$36 T$, no obstante, recogía sin dudas "sor" en su ejemplar.

37 Cf., por ejemplo, Paolo Chiesa, Elementi di critica testuale, Bologna, Pàtron, 2012², p. 117: "è molto difficile che un copista contamini introducendo un errore patente".

38 Tampoco cabe pensar que $A$, donde comparte con $C T$ la lección genuina, se haya emendado ope ingenii.

39 Los reagrupamientos en el seno de $\beta$ varían, naturalmente, dependiendo de la presencia o la ausencia de los testimonios en ese determinado punto del texto.

40 Y ello corresponde, en efecto, a la observación de Paolo Chiesa citada en la nota 37.

41 El simple reagrupamiento $A E$, donde, en representación de $\beta$, figuran solo estos dos testimonios, se excluye. No es posible determinar, en tal caso, si la lección errónea remite a $\beta$ o a su descendiente $\gamma$. 
res comunes a $V A G$ : v. $1227^{42}$. Errores comunes a $V A$ : vv. $2031,5058^{43}$, 5276.

Micha no contempla el caso de reagrupamientos $\alpha A$ contra $\beta^{44}$. Sin embargo, señala un buen número de coincidencias de $C A$ y $T A$ contra $T / C+\beta$, presuponiendo en todo momento, aunque sin llegarla a demostrar, la relación $C \rightarrow A$ y $T \rightarrow A$. Estudiemos, por lo tanto, estos vínculos partiendo de su análisis.

Acuerdos $C A$ por lo que concierne a los errores ${ }^{45}$. La prueba principal adoptada por Micha atańe a los vv. 1139-43, y en particular a 1140 y 1142. Aporto a continuación el texto crítico de Foerster (hasta los vv. 1144-45) con las variantes de la tradición que afectan a esta cuestión:

Et bote anz le col et la teste $\mid$ Et esgarde a mont vers le feste, $(C$ par la fenestre $A$ vers la fenestre) | Si voit deus espees venir. | Arriers se tret, et retenir ( $C$ Adonc se prist a $A$ Lors se reprist a $E$ Lors se conmenche a) | Li chevalier lor cos ne porent: $(A$ L. ch. coissir n. p. $) \mid$ De tel äir mëuz les orent ( $C$ Detenir qu'esmeüz l. o.) $\mid$ Q'an terre les espees fierent $[\ldots]$

Observa Micha que la rima "teste: fenestre", de $C A$ ) parece ajena al uso del autor ${ }^{46}$ (y, yo añadiría, "fenestre" es lectio facilior) y que la sintaxis de los vv. 1142-43 en $C A$ es inaceptable (aunque también lo es en $E$, incluso mostrando un verbo diferente). En el 1140 la sustitución del difficilior "feste" por "fenestre" podría ser poligenética; quizás menos probable la

$42 E$ se une aquí a $C T$, quizás también por contaminación.

43 También en estos dos versos $E$ se une a $C T$.

44 Ya hemos dicho anteriormente que el filólogo francés no reconoce la existencia de la rama $\alpha$ de Foerster.

45 Alexandre Micha, op. cit., p. 140.

46 Aunque las rimas de este tipo no sean raras en la versificación francesa medieval: cf. Jacques Chaurand, Introduction à la dialectologie française, Paris, Bordas, 1972, p. 94: "Les rimes où un $\mathrm{R}$ soit implosif, soit explosif devant $e$ final ne compte pas sont en usage un peu partout"; el autor cita, del Chevalier de la Charrette, además de este ejemplo, también el de los vv. 1883-84, donde $C$ presenta la rima "trueve": "uevre" ( $T$ "trueve": "muele"; VAE [= $\beta]$ "troeve": "noeve"; $A$ carece del final del v. 1883); se trata de una lectio singularis de C, además inferior por el sentido; pero abre la puerta a la posibilidad de un encuentro fortuito entre $C$ y $A$ en los vv. 1139-40: para Guiot la rima es aceptable. 
sustitución en el v. 1142, que, en gran parte, comparte $E$ y produce efectos distintos en sus testimonios en los versos siguientes ${ }^{47}$. El acuerdo no parece del todo significativo ${ }^{48}$.

Micha añade en una nota, sin comentarios, algunos acuerdos de $C A$ en errores ("fautes") y variantes adiáforas ("leçons") 49 . De las "fautes" se deben descartar las de los vv. 1766, 2220, 2692, 4659 $9^{50}$.

Queda la del v. 2201: Foerster "Andormiz s'est; talant an ot"; $C$ "Si dormi se talant en ot" $A$ "Si dormi se talent en ot" $\| T$ "Endormiz s'est talent en ot" $\| V$ "Et dormir se talent en ot" $E$ "Et dormir se talent enn out" ( $V D$ "et il s'endormy, se talent en ot"). La lección de $T$, recogida por Foerster, está completamente aislada y no es aceptable en el plano del contenido $^{51}$. Quedan, por lo tanto, $C A$ por una parte y $V E(=\beta)$ por otra. Su oposición se reduce a $C A$ "Si dormi" vs. "Et dormir" $V E$; el resto del verso es perfectamente idéntico. "Et dormir" $V E$ sería probablemente la lección que se debería acoger en el texto en tanto en cuanto enlaza sintácticamente con el verso anterior y determina un fuerte encabalgamiento, típico del estilo de Chrétien; además "Et dormir" explica "Endormiz" $T$ mucho más satisfactoriamente que "Si dormi" $C A$. La lección de $C A$ es

47 En particular, es interesante la modificación de $C$, que en el v. 1144 cambia "De tel aïr" por "De tenir", dando a "porent", v. 1143, el infinitivo dependiente que necesitaba y sin el cual la frase no tiene sentido. $A$ intenta resolver torpemente el problema del v. 1143, introduciendo el improbable infinitivo "coissir" en lugar de "lor cos".

48 Se debe señalar la poco creíble defensa de la lección de $C A$ por parte de Mario Roques: Les Romans de Chrétien de Troyes, Édités d'après la copie de Guiot (Bibl. nat. fr. 794), III: Le Chevalier de la Charrete, ed. Mario Roques, Paris, Champion, 1983, p. 222, nota al v. 1128. Entre los editores posteriores a Foerster, Roques es el único que recoge íntegramente la lección de $C$.

49 Alexandre Micha, op. cit., p. 140, n. 1.

$50 \mathrm{El}$ primer acuerdo no es significativo; en el resto de casos, o el acuerdo no subsiste o la lección es unánime.

51 El razonamiento de Foerster (Christian von Troyes, op. cit., p. 380) es curioso, a la par que bastante ingenuo, basado, como está, en peregrinos criterios psicológicos: $T$ tiene razón porque Lancelot está cansado debido a las fatigas de la jornada; y por otra parte, alguien como él, que ha dormido sin problemas en el lecho maravilloso, no puede dejar de dormir profundamente también en casa del "vavassor". Por consiguiente, poner en entredicho sus ganas de dormir (como hacen, incluso con lecciones diferentes, $C V A E$ ), carecería de sentido. Este es uno de los casos en los que el editor alemán no tiene en cuenta el stemma que él mismo traza. 
una trivialización carente de valor conjuntivo.

Entre las leçons se deben descartar las de los vv. 545, 648, 2530, 3074 (acuerdos claramente casuales), y las de los vv. 1382, 2611 (acuerdos inexistentes).

En el v. $728 A$ (que coincide solo en parte con $C$ ) conserva probablemente la lección original en oposición a una innovación de $T E^{52}$ : $C A$ "Qu'il n'ot ne voit ne rien ( $A$ si) n'antant" $\| T E$ "Que il ne voit ne il n'entent".

En el v. $2072 C A$ "trestuit se baillent" se opone a "tuit (mout $V$ ) se travaillent" $T V$ (también a $V D$ "moult se travaillent" $)^{53}$.

En el v. 3759 a $T$ "si pres li venoit" se oponen por una parte $V G$ "si pres la voit" ( $G$ "le veoit") y por otra $C A$ "si pres l'i $(A$ le $)$ menoit"; Foerster acoge en el texto la lección de $T$, pero la de $C A$ es igualmente aceptable e, incluso, tal vez más fiable ${ }^{54}$.

En el v. 5689 a TFE "Ne por morir" (aceptado por Foerster) se oponen $C A$ "Por a morir", mientras que $V$ presenta una lectio singularis; la construcción de $C A$ ('a riesgo de morir') está atestiguada, pero difícilmente encaja en el complejo período.

En resumen, y una vez eliminados los casos que carecen de valor, en los vv. 2072 y 5689 el acuerdo de $C A$ se manifiesta en lecciones que no son erróneas, sino probablemente inferiores; en el v. $728 C A$ parecen presentar la lección genuina.

Se pueden enumerar otros once casos de acuerdos de $C A$ contra $T+$ $\beta^{55}$, algunos de los cuales tienen que ver con lecciones inferiores, ninguno con errores manifiestos y casi ninguno ajeno a la sospecha de la poligénesis. La única, aunque relevante, excepción deriva del pasaje de los vv.

52 El tricolon de CA ("ot", "voit", "antant") tiene a su favor algunos loci paralleli en otros romans de Chrétien.

$53 E$ modifica erróneamente: "mont se penoient", que resulta hipermétrico y contraviene. La construcción "se baillier", aunque rara, está atestiguada, si bien parece ajena al usus del autor.

54 La similitud gráfica entre $T$ y $C A$ ("l'i venoit"/ "l'i menoit") es tal que llega a privar de valor al acuerdo.

55 Dejando al margen tanto un gran número de acuerdos de $C A$, claramente insignificantes, como los casos de oposición $C A / T E$, a falta de otros testimonios, ya que $E$ también sería susceptible de considerarse contaminado por un códice $\alpha$, probablemente cercano a $T$. 
5488-90, donde podría estar implicada una lección de arquetipo.

v. 158: $C A$ “Otroit ( $A$ Li o.) ce qu'il ( $A$ que) voldra" $\| T E G$ "Otroit ce qu'il dira"; la lección de $C A$ ("voldra") es inferior (cf. el v. 169), pero no errónea; sin embargo, la sustitución "dira" $\rightarrow$ "voldra” no escapa a la sospecha de poligénesis, sobre todo en presencia de "otroit" ('conceda').

v. 894: $C A$ "li vient sus" || TVE "li cort sus"; tanto "venir sus" como "corir sus" se usan y están atestiguados en el autor con el significado de 'asaltar'; las sustituciones individuales de una expresión por otra son demasiado fáciles para que el acuerdo resulte significativo.

v. 970: $C A$ "tristesce" || TVEG "destrece"56; "tristesce" es claramente inferior, pero, en este contexto, podría ser poligenético.

v. 1039: $C$ "s'asiet" $A$ "s'i asiet" $\| T V E$ "li siet" $\| \neq G ; C A$ invierten las palabras en rima en los vv. 1039-40, resultando inferiores por el sentido; pero la innovación, que en los dos testimonios produce repercusiones diferentes en el segundo verso, no es significativa.

vv. 3615-3616: $C A$ "peitrax... | Estriés" || TVE "estri... | Poitral”"57; es probable que el orden de $T(V) E$, mejor documentado, sea el original; pero la inversión de $C A$ podría ser perfectamente fortuita.

v. 4291: $C A$ "gole" || TV "gorge"58 || $E$ "col"; si bien "gorge" es, desde luego, preferible (cf. los vv. 4324, 4328), "gole" y "col” son sinónimos.

v. 4303: $C$ "met le laz antor sa teste" $A$ "met entor le lac sa teste" || TVE "mest parmi le laz sa teste" ${ }^{59}$; $C A$ anticipan el concepto "antor" del verso siguiente ("Tant qu'antor le col li areste"); $A$ de manera tan torpe que priva de sentido a la expresión.

v. 4405: CA "enor" || TVE “amor"; "amor” es claramente erróneo (cf. el v. 4404), pero se tratará de una banalización independiente; la hipótesis alternativa es mucho más onerosa y francamente improbable: "amor", error de arquetipo, corregido por $C$ y $A$ de manera independiente, o bien por un ascendente de $C$, del que lo habría tomado A por contaminación.

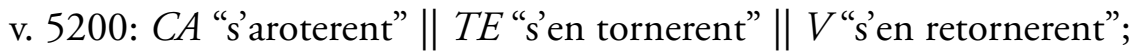

56 "Destrece" se encuentra también en $V D$.

57 En el v. $3516 V$ cambia radicalmente.

58 Compartido por $V D$.

59 También VD: "mist parmy le laz sa teste". 
la lección de $C A$ es claramente diffcilior ${ }^{60}$; TVE banalizan independientemente.

v. $5488 C A$ "peor" || TVE "pooir"; vv. 5489-90 VE om.; "pooir" de $T V E$ es erróneo e insensato; la falta en $V E$ de los vv. 5489-90, que dependen sintácticamente de "peor" y que con "pooir" carecen de sentido, es síntoma evidente de la conciencia del error y un ineficaz intento de remediarlo; la laguna debería remitir a $\beta$, colmada por $A$ por contaminación con un ascendente de $C$. Por lo tanto, "pooir" será un error de arqueti$\mathrm{po}^{61}$, corregido ope ingenii por un ascendente de $C$; a este se habrá unido $A$ en "peor" y en los versos de los que $\beta$ carece. $T$, en cambio, reproduce el arquetipo con su falta de sentido.

vv. 5789-90: $C A$ "porterent: erent" || TVF "portoient: estoient"; la rima de $C A$ podría parecer, a simple vista, difficilior, pero en un análisis más profundo se revela contraria al probable usus del autor ${ }^{62}$, si bien no errónea en modo abstracto. Así las cosas, no se puede excluir que la rima de $C A$ se encontrase en el arquetipo y haya sido 'corregida' ${ }^{63}$ independientemente por TVF; la lección $C A$ no es separativa.

Micha también identifica algunas convergencias entre $T$ y $A^{64}$. Discute los casos de los vv. 1136, 1513 e 4571, relegando a una nota ${ }^{65}$ algunos acuerdos más.

En el v. 1136 tenemos $C V E$ "de covant" ( $V$ "covent" $E$ "convent") \|

60 Para este verbo (que con uso reflexivo se atestigua solo dos veces en toda la obra de Chrétien), cf. la nota de Foerster (Christian von Troyes, op. cit., p. 407).

61 Parece excluida, esta vez, la poligénesis en TVE, a pesar de la cercanía gráfica de las dos palabras. Igualmente improbable se presenta la hipótesis de una corrección ope ingenii por parte de $C$ y de $A$ de manera independiente. Por lo demás, los dos versos que faltan en $\beta$, no podían ser inventados por $A$. "Pooir" por "peor" es un error conjuntivo, pero no separativo.

62 Cf. Maurizio Perugi, "Patologia testuale e fattori dinamici seriali nella tradizione dell'Yvain di Chrétien de Troyes", Studi Medievali, XXXIV, 2 (1993), pp. 841-60; sobre todo pp. 852-53, donde se subraya la tendencia de la tradición a "censurar" las formas etimológicas del imperfecto y del futuro de estre, en aras de aquellas no etimológicas, que han prevalecido. Sin embargo, en otros dos pasajes de los romans de Chrétien, (i)erent imperfecto se encuentra en posición de rima con-ierent, y no con-erent como aquí.

63 O sea, satisfactoriamente 'banalizada'.

64 Alexandre Micha, op. cit., pp. 137-38.

65 Ibidem, p. 138, n. 1. 
$T A$ "doucement"; aunque "doucement" sea poco adecuado al contexto y destruya la rima leonina "sovant": "covant", el acuerdo TA podría perfectamente ser casual: "decouēt" e "doucemēe" (quizás "doucemt", con una barra sobre la $m$ para abreviar en) son paleográficamente bastante cercanos.

En el v. 1513 la convergencia es inexistente.

En el v. 4571 tampoco se observa una correspondencia exacta entre $T$ y $A$ : $C$ "Se fet las et se fet couchier" \| $T$ "Ne se coucha ne fist couchier" || $V$ "Le dit atant se vet couchier" || $A$ "Se couca et si fist coucier" $\| E$ "Dist icen et s'ala couchier"; como se ve, las lecciones de $T$ y $A$ no son en absoluto asimilables; estamos, más bien, ante la presencia de una difracción, en la que cada testimonio opera por su cuenta ${ }^{66}$; no obstante, Micha se atreve a afirmar que "la source commune [a $T A]$ est assurée" ${ }^{\prime 67}$.

Los casos relegados a nota son mucho más numerosos:

\section{"Fautes"}

En el v. 2680 el acuerdo de $T A$ se produce sobre una lección claramente errónea, pero es parcial y no excluye la sospecha de poligénesis ${ }^{68}$.

En el v. 3359 el acuerdo es inexistente.

En el v. 3431 "plus", común a $T A$, anticipa el verso siguiente.

En el v. 5076: $T A$ deben haber conservado la lección original, banalizada por $C V E^{69}$.

\section{"Leçons"}

vv. 785, 1658: banalizaciones evidentes por parte de $T A$.

vv. 1067, 2034, 2412, 2478, 2665-66, 3963: acuerdos de TA muy probablemente casuales.

66 Y donde $V$ podría haber conservado la lección original: "Le dit" está sintácticamente vinculado a "por la gent", v. 4570, con un fuerte encabalgamiento, muy típico del estilo del autor.

67 Alexandre Micha, op. cit., p. 138.

$68 T$ "que ce fust .i. cuens" $A$ "qu'il doie estre quens" $\| C V E G$ "qu'il doie estre suens" $(E$ "que devoit" $G$ "que doie"). $A$ conserva "doie", ciertamente original, mientras que $T$ lo modifica con "ce fust".

69 Que, en efecto, muestran lecciones distintas. 
v. 1919: el acuerdo de $T A$ es parcial $^{70}$, y es muy probable que la lección de $T$ sea original.

v. 3323: el acuerdo $T A$ debe ser extendido a $E$.

v. 3822: acuerdo $T A$ inexistente.

Entre las "leçons", vale la pena discutir dos casos: vv. 3864, 5007-08.

v. 3864: CVG "tochast" || TA "tornast" es, sin duda, lección inferior, aunque no se trata de un error manifiesto; además, la similitud gráfica disminuye notablemente su valor.

vv. 5007-5008: $C V E$ "Qant li seiremant furent fet" || TA "Et quant les seremenz ont faiz" ( $A$ "Quant $[\ldots]$ orent $[\ldots]$ "); CVE "Lor cheval ( $V$ Et li ch. E Et lors ch.) lor furent fors tret" $\| T A$ "Lors orent les chevaux fors traiz"; $C V E$ muestran una construcción pasiva, mientras que $T A$ una activa; variantes adiáforas y acuerdos poco significativos.

Se pueden citar otros acuerdos $T A$ contra $C+\beta$, pero de escaso valor ${ }^{71}$.

v. 1258: TA "plus rien ne li querroit" $\| C V E G$ "plus ne li requerroit"; $T A$, no habiendo entendido que "plus" es objeto de "requerroit", insertan "rien", acortando la forma verbal por razones métricas; el acuerdo no es de los más significativos.

v. 1536: TA “ainz m'ocirroie" || $C V E G$ "einz me (VE en $G$ i) morroie"; la lección genuina parece conservada por $V E$ ("en morroie"); la construcción de $C$, en su sentido literal, es ajena a la obra de Chrétien; en vista del contexto, $T A$ “m'ocirroie" podría ser una coincidencia casual.

v. 1578: TA "Ne tant de los" || CVEG "Ne tant d'enor"; "enor" y "los" son sinónimos; el acuerdo de $T A$ sobre "de los" no es de los más significativos.

v. 2045: TA "Et je en sui" || $C V E$ "Et g'en ( $V$ ge) resui”; "je en sui” de $T A$ podría ser prosódicamente difícil por la ausencia de elisión de "je" ante "en"; "resui" de CVE ('a mi vez, yo también soy') es más difícil (y más adecuado) sobre la base del sentido; la oposición TA / CVE no parece, tampoco en este caso, significativa.

v. 2478: TA “en soupeçon" || $C V E$ "an cusançon"; "cusançon” es, desde luego, más adecuado al contexto, pero "sospeçon” se insinúa, como alter-

$70 T$ "Li estrange prison i tienent" $\| A$ "Li estrange prison i sont".

71 Excluidos, con más motivos, los habituales y numerosos encuentros claramente casuales. 
nativa a "cusançon", en la tradición del Chevalier au Lion, v. 108: $H$ "sospeçon" VFAS “cusençon" $G$ "encusençon" $R$ "marison" 72 ; $T$ y podrían haberse encontrado de modo casual.

vv. 2665-66: TA "seoit": "servoit" \| CVEG "seoient": "servoient"; la tercera persona del singular de $T A$ es errónea (cf. sobre todo el v. 2669), pero probablemente poligenética; el pasaje parece pensado a propósito para confundir a los copistas; y de hecho en los vv. 2667-68, $A$ pasa a la tercera del plural ("meïssent": "preïssent"), mientras que $T$ mantiene la tercera del singular, en la que coincide con $V$ ("meïst": "preïst"); todo ello con modificaciones y adaptaciones individuales.

vv. 4327-4328: TA "toutes": "routes" || CVE "rotes": "totes"; se trata del orden de las palabras en rima; las alternativas son equivalentes y sospechosas de poligénesis.

El análisis de Micha, desprovisto de la nutrida serie de coincidencias insignificantes o inexistentes, e incrementado con el resto de los casos seńalados, se revela muy escaso. Se salvan, quizás, por lo que respecta a $C A$, los vv. 1139-43, 2201, 5869. Solo el acuerdo en los vv. 5488-90, donde probablemente haya implicado un error de arquetipo, parece altamente significativo y, por ende, decisivo. En cambio, ningún acuerdo $T A$ contra $C+\beta$ resulta significativo tras un examen detenido.

Sobre la base del material hasta aquí recogido, la conclusión es que $A$, sobre un ejemplar base perteneciente a $\beta$, ha injertado una serie de lecciones provenientes de $\alpha$, en particular, de un testimonio de control afín a $C$. Una línea discontinua debería, por tanto, trazarse en el esquema de Foerster entre $A$ y un ascendente de $C(C)$.

Hasta ahora hemos hablado, por comodidad, de $A$ como actor de un proceso de contaminación entre una base que remite a $\beta$ y un ejemplar de control del área $\alpha$ (cercano a $C$ ). Pero el códice de Chantilly no es, desde luego, el lugar en que la contaminación se verifica de modo efectivo.

Ante todo, no es posible identificar rastro alguno de tal actividad. No hay variantes interlineares o marginales, nada que pueda llevar a pensar

72 Christian von Troyes, Der Löwenritter (Yvain), ed. Wendelin Foerster, Halle, Niemeyer, 1887. La varia lectio se ha extraído de la transcripción sinóptica de todos los manuscritos, al cuidado de Kajsa Meyer, disponible en la web http://www.lfa. uottawa.ca/activites/textes/kmeyer/kpres.html. El manuscrito denominado $H$ corresponde con nuestro $C$. 
en una responsabilidad del copista de nuestro códice. Además, como se ha dicho, el texto de $A$ es mútilo y concluye en el v. 5873. Es altamente improbable que el escriba de $A$ tuviese a su disposición dos ejemplares y que ambos careciesen de la parte conclusiva del roman ${ }^{73}$.

La contaminación debió de producirse a nivel de un antecedente de $A$. En tal caso, la citada línea discontinua debería trazarse, para mayor precisión, entre este antecedente $\left(A^{\prime}\right)$ y $\alpha$, o entre $A^{\prime}$ y el ascendente $C^{\prime}$ de $C$.

Creo que las observaciones brindadas en este estudio refuerzan e incluso enriquecen la reconstrucción de Foerster. En este caso, tal vez particularmente favorable, la contaminación, una vez sacada a la luz y bien circunscrita, tiene el efecto, paradójico solo aparentemente, de volver más límpidas las aguas que, por su causa, corrían el riesgo de verse enturbiadas. El estudio tipológico de los fenómenos poligenéticos en las tradiciones de los romans en francés antiguo facilitaría análisis como este, favoreciendo la eliminación de una gran masa de materiales superfluos ${ }^{74}$. Más aún, sería extremadamente útil, si bien de ardua compilación, un diccionario de Chrétien de Troyes que recogiese, para cada lema, las variantes que ofrece la tradición.

73 Recuérdese que la mutilación no se debe, en $A$, a causas materiales (pérdida de folios o fascículos): el texto se detiene ex abrupto alrededor de la mitad de la columna $a$ del folio 213v; el resto del mismo permanece en blanco.

74 Un sugestivo intento en esta dirección y aplicado a la Commedia dantesca puede verse en Caterina Brandoli, "Due canoni a confronto: i luoghi di Barbi e lo scrutinio di Petrocchi", en Nuove prospettive sulla tradizione della "Commedia". Una guida filologico-linguistica al poema dantesco, ed. Paolo Trovato, Firenze, Franco Cesati, 2007, pp. 99-214. 